\title{
Prolonged duration of response in lenvatinib responders with thyroid cancer
}

\author{
Andrew G Gianoukakis,2, Corina E Dutcus³, Nicolas Batty33, Matthew Guo³ and Mahadi Baig³ \\ 'Los Angeles Biomedical Research Institute and Division of Endocrinology and Metabolism, Department of Medicine at Harbor-UCLA Medical Center, \\ Torrance, California, USA \\ 2David Geffen School of Medicine, University of California - Los Angeles, Los Angeles, California, USA \\ ${ }^{3}$ Eisai Inc., Woodcliff Lake, New Jersey, USA \\ Correspondence should be addressed to A G Gianoukakis: agianoukakis@mednet.ucla.edu
}

\begin{abstract}
We present an updated analysis of lenvatinib in radioiodine-refractory differentiated thyroid cancer (RR-DTC) with new duration of response (DOR) data unavailable for the primary analysis. In this randomized, double-blind, multicenter, placebo-controlled phase 3 study, patients $\geq 18$ years old with measurable, pathologically confirmed RR-DTC with independent radiologic confirmation of disease progression within the previous 13 months were randomized 2:1 to oral lenvatinib $24 \mathrm{mg} /$ day or placebo. The main outcome measures for this analysis are DOR and progression-free survival (PFS). The median DOR for all lenvatinib responders (patients with complete or partial responses; objective response rate: $60.2 \%$; 95\% confidence interval (CI) 54.2-66.1) was 30.0 months (95\% Cl 18.4-36.7) and was generally similar across subgroups. DOR was shorter in patients with greater disease burden and with brain and liver metastases. Updated median PFS was longer in the overall lenvatinib group vs placebo (19.4 vs 3.7 months; hazard ratio (HR) $0.24 ; 99 \% \mathrm{Cl} 0.17-0.35$; nominal $P<0.0001)$. In lenvatinib responders, median PFS was 33.1 months $(95 \% \mathrm{Cl} 27.8-44.6)$ vs 7.9 months $(95 \% \mathrm{Cl} 5.8-10.7)$ in nonresponders. The median DOR of 30.0 months seen with patients who achieved complete or partial responses with lenvatinib $(60.2 \%)$ demonstrates that lenvatinib responders can have prolonged, durable and clinically meaningful responses. Prolonged PFS (33.1 months) was also observed in these lenvatinib responders.
\end{abstract}

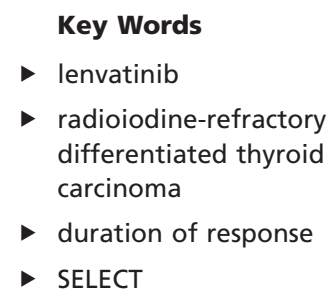

Endocrine-Related Cancer (2018) 25, 699-704

\section{Introduction}

Differentiated thyroid cancer (DTC) is the most common type of thyroid cancer in the United States, where papillary and follicular thyroid carcinomas account for up to $94 \%$ of all thyroid carcinoma cases (Busaidy \& Cabanillas 2012). The standard treatment for most patients receiving diagnoses of DTC is surgery followed by administration of radioactive iodine (Cooper et al. 2009). However, approximately $10-15 \%$ of cancers become refractory to radioiodine treatment, which is then referred to as radioiodine-refractory DTC (RR-DTC) (Busaidy \& Cabanillas 2012, Pacini et al. 2012). For these cases, the life expectancy is 3-6 years and the 10-year survival rate is $10 \%$ from the time of metastatic detection (Durante et al. 2006, Pacini et al. 2012, Xing et al. 2013). Patients with RR-DTC have few treatment options and typically require alternative therapies (Pacini et al. 2012).

Tyrosine kinase inhibitor (TKI) therapy is a recently approved option for patients with RR-DTC. The first
2018 The authors Published by Bioscientifica Ltd. Printed in Great Britain

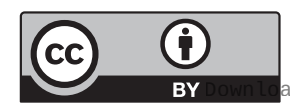

This work is licensed under a Creative Commons Attribution 4.0 International License. 
targeted agent shown to improve progression-free survival (PFS) in patients was the multikinase inhibitor, sorafenib (Brose et al. 2014), approved for use in patients with RR-DTC in 2013 by the US Food and Drug Administration (FDA) (Worden 2014). More recently, based on the results of the randomized, double-blind, multicenter, phase 3 Study of (E7080) Lenvatinib in Differentiated Cancer of the Thyroid (SELECT), the FDA approved lenvatinib for the treatment of locally recurrent or metastatic, progressive RR-DTC (Schlumberger et al. 2015). Lenvatinib is an oral, multikinase inhibitor of vascular endothelial growth factor (VEGF) receptor (VEGFR) 1-3, fibroblast growth factor receptor (FGFR) 1-4, platelet-derived growth factor receptor alpha (PDGFR $\alpha$ ), ret proto-oncogene (RET) and stem cell factor receptor (KIT) (Matsui et al. 2008a,b, Okamoto et al. 2013, Tohyama et al. 2014, Yamamoto et al . 2014). In contrast to sorafenib, lenvatinib targets FGFR in addition to VEGFR, which is thought to be important for preventing the development of resistance to TKI therapies as the FGFR pathway offers an intracellular alternative to the VEGFR pathway (Laursen et al. 2016).

In the primary analysis of SELECT, lenvatinib was shown to significantly prolong PFS compared with placebo (18.3 vs 3.6 months; hazard ratio (HR) 0.21; 99\% confidence interval (CI) $0.14-0.31 ; \quad P<0.001$ ) (Schlumberger et al. 2015). At the time of the primary analysis, the median duration of overall response (DOR) had not been reached. Here, we report updated analyses of lenvatinib efficacy in SELECT with an emphasis on DOR.

\section{Materials and methods}

\section{SELECT}

The primary analysis of SELECT, a phase 3, randomized, placebo-controlled study, was previously reported (Schlumberger et al. 2015). Eligible patients were $\geq 18$ years of age and had measurable, pathologically confirmed DTC, evidence of radioiodine-refractory disease, and independently reviewed radiologic evidence of progression within the previous 13 months. Patients were permitted to have received up to 1 prior treatment with a TKI. Patients were randomly assigned 2:1 to receive $24 \mathrm{mg}$ of oral lenvatinib daily or placebo until disease progression, development of unacceptable toxicity or withdrawal of consent. The data cutoff for the primary analysis was November 15, 2013, after which the study continued with an open-label phase in which patients in the lenvatinib group could remain on therapy and those in the placebo group with progressive disease could choose to receive lenvatinib treatment. Patients in the placebo group who did not choose to receive treatment with lenvatinib $(n=22)$ in the open-label phase of the study were followed for survival. All patients enrolled in SELECT provided written informed consent. The study protocol was approved by the relevant institutional review bodies, and the study was conducted in accordance with the Declaration of Helsinki and local laws.

\section{Efficacy analyses}

The data cutoff for this updated analysis was September 1, 2016. Tumors used in this updated analysis were assessed by clinical trial investigators. Responders were defined as patients who had a complete response (CR) or partial response (PR) as their best overall response per Response Evaluation Criteria in Solid Tumors v1.1. DOR was examined for lenvatinib-treated patients who had PR or CR overall and by patient subgroup (age, sex, tumor subtype, baseline disease burden, baseline Eastern Cooperative Oncology Group performance status, metastasis site or prior VEGF therapy). Exploratory efficacy endpoints included objective response rate (ORR), disease control rate (DCR) and clinical benefit rate (CBR). ORR was defined as the proportion of patients with best overall response of CR or PR. DCR was identified as the proportion of patients with a best overall response of CR or PR or stable disease (SD; achieved $\geq 7$ weeks after Day 1 of the open-label treatment period). CBR was defined as the proportion of subjects who had a best overall response of CR or PR or durable SD (of duration $\geq 23$ weeks).

In SELECT, the primary endpoint was PFS, which was assessed overall, including patients who did or did not respond to treatment. PFS is defined as the time from randomization until either objective tumor progression or death. Median PFS was estimated and plotted using the Kaplan-Meier method, and 95\% CIs were constructed with a generalized Brookmeyer and Crowley method. HRs are estimated from a Cox proportional hazards model stratified by randomization factors. For overall PFS, the HR is expressed for lenvatinib and placebo, and for responder analysis, HR is expressed for responders and non-responders.

\section{Results}

Median duration of response of $\mathbf{3 0}$ months in responders

This updated analysis showed that patients who responded to lenvatinib (achieved CR or PR) could have prolonged, durable responses. The median DOR for all patients who

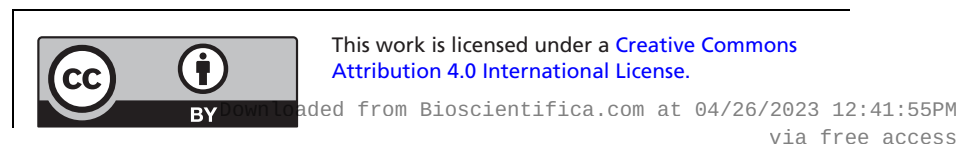


Table 1 Median DOR for the lenvatinib treatment group in all responders and by subgroup.

\begin{tabular}{|c|c|c|}
\hline Subgroup & $n$ & $\begin{array}{c}\text { Median DOR; } \\
\text { lenvatinib treatment } \\
\text { group; months }(95 \% \mathrm{Cl})\end{array}$ \\
\hline All responders & 157 & $30.0(18.4-36.7)$ \\
\hline \multicolumn{3}{|l|}{ Age (years) } \\
\hline$\leq 65$ & 104 & $27.5(14.7-36.7)$ \\
\hline$>65$ & 53 & $31.3(18.4-43.5)$ \\
\hline \multicolumn{3}{|l|}{ Sex } \\
\hline Male & 73 & $30.0(16.8-43.5)$ \\
\hline Female & 84 & $27.3(16.8-43.3)$ \\
\hline \multicolumn{3}{|c|}{ Baseline diseases burden (mm) } \\
\hline$\leq 35$ & 37 & 44.3 (30.5-NE) \\
\hline $35-60$ & 45 & $27.5(12.9-45.7)$ \\
\hline $60-92$ & 38 & $18.0(11.0-35.0)$ \\
\hline$>92$ & 37 & $15.7(11.1-35.2)$ \\
\hline \multicolumn{3}{|c|}{ Bone metastasis only } \\
\hline Yes & 1 & NE (NE-NE) \\
\hline No & 156 & $29.9(18.4-36.7)$ \\
\hline \multicolumn{3}{|l|}{ Lung metastasis } \\
\hline Yes & 141 & $29.9(17.5-37.8)$ \\
\hline No & 16 & 34.0 (7.4-NE) \\
\hline \multicolumn{3}{|l|}{ Liver metastasis } \\
\hline Yes & 24 & 15.7 (3.7-NE) \\
\hline No & 133 & $30.5(22.2-41.4)$ \\
\hline \multicolumn{3}{|l|}{ Brain metastasis } \\
\hline Yes & 5 & $9.3(0.9-13.8)$ \\
\hline No & 152 & $30.5(22.2-41.4)$ \\
\hline \multicolumn{3}{|c|}{ Lymph node target lesions } \\
\hline Yes & 75 & $27.2(12.9-35.2)$ \\
\hline No & 82 & 30.5 (22.2-NE) \\
\hline \multicolumn{3}{|c|}{ Prior VEGF therapy } \\
\hline Yes & 40 & $29.9(7.5-45.7)$ \\
\hline No & 117 & $30.0(18.4-43.3)$ \\
\hline \multicolumn{3}{|c|}{ Baseline tumor subtype } \\
\hline Papillary & 99 & $29.9(16.8-43.3)$ \\
\hline Follicular & 58 & $30.0(15.7-45.7)$ \\
\hline \multicolumn{3}{|c|}{ Baseline ECOG PS } \\
\hline 0 & 102 & $31.3(18.4-43.5)$ \\
\hline 1 & 52 & $27.5(13.3-36.7)$ \\
\hline$>1$ & 3 & $11.1(0.9-11.1)$ \\
\hline
\end{tabular}

Updated data, cutoff: 1 September 2016

$\mathrm{Cl}$, confidence interval; DOR, duration of response; ECOG PS, Eastern Cooperative Oncology Group performance status; NE, not evaluable; VEGF, vascular endothelial growth factor.

responded to lenvatinib treatment was 30.0 months (95\% CI, 18.4-36.7). A summary of the median DOR for all responders and by patient subgroup is shown in Table 1. Median DOR in lenvatinib-treated patients was generally similar by patient subgroup, but appeared to be shorter in patients with greater disease burden (tumor size $\leq 35 \mathrm{~mm}: 44.3$ months; 35-60 mm: 27.5 months; 60-92 mm: 18.0 months; >92 mm: 15.7 months), patients with liver metastasis (yes: 15.7 months; no: 30.5 months) and patients with brain metastasis (yes: 9.3 months; no: 30.5 months). Median DOR was similar between responders who had received one prior VEGF therapy (29.9 months) and those who had not received prior VEGFR therapy (30.0 months).

\section{Updated progression-free survival}

In SELECT, 261 (male: 125, female: 136) patients were enrolled to receive lenvatinib and 131 (male: 75, female: 56) patients received placebo treatment. The updated analysis showed a prolonged median PFS of 19.4 months in the lenvatinib group compared with 3.7 months in the placebo group (HR 0.24; 99\% CI, 0.17-0.35; nominal $P<0.0001$; Fig. 1). As of the updated data cutoff, $80.8 \%$ of lenvatinib-treated and $9.9 \%$ of placebo-treated patients from SELECT experienced grade $\geq 3$ treatmentrelated treatment-emergent adverse events, and no new treatment-related deaths had occurred.

\section{Median progression-free survival of $\mathbf{3 3 . 1}$ months in responders}

In the lenvatinib-treated group, the median PFS in patients who demonstrated a CR or PR was 33.1 months (95\% CI, 27.8-44.6, Fig. 2), whereas in patients who did not show CR or PR, the median PFS was 7.9 months (95\% CI, 5.8-10.7, Fig. 2).

The tumor response according to investigator assessment for this updated analysis is summarized in Table 2. The ORR was $60.2 \%$ (95\% CI, 54.2-66.1) for lenvatinib-treated patients compared with 2.3\% (95\% CI, 0.0-4.9) for placebo-treated patients. Of note, since the primary analysis, 1 additional patient achieved a CR in both treatment groups. The DCR was $90.4 \%$ in the lenvatinib group compared with $61.1 \%$ in the placebo group $(P<0.0001)$, and the CBR was $82.0 \%$ for lenvatinib and $41.2 \%$ for placebo. Median time to first objective response was 3.5 months (95\% CI, 1.9-3.7 months) in the lenvatinib group as evaluated by investigator assessment.

\section{Discussion}

The durability of the responses observed measured by median DOR could not be estimated in the original analysis of lenvatinib in patients with RR-DTC from SELECT (Schlumberger et al. 2015). However, this updated analysis showed that patients who responded to lenvatinib continued to have prolonged, durable responses, lasting a median of 30.0 months. This prolonged DOR was observed across several patient subgroups and was not influenced by age, sex or tumor subtype. However, some 


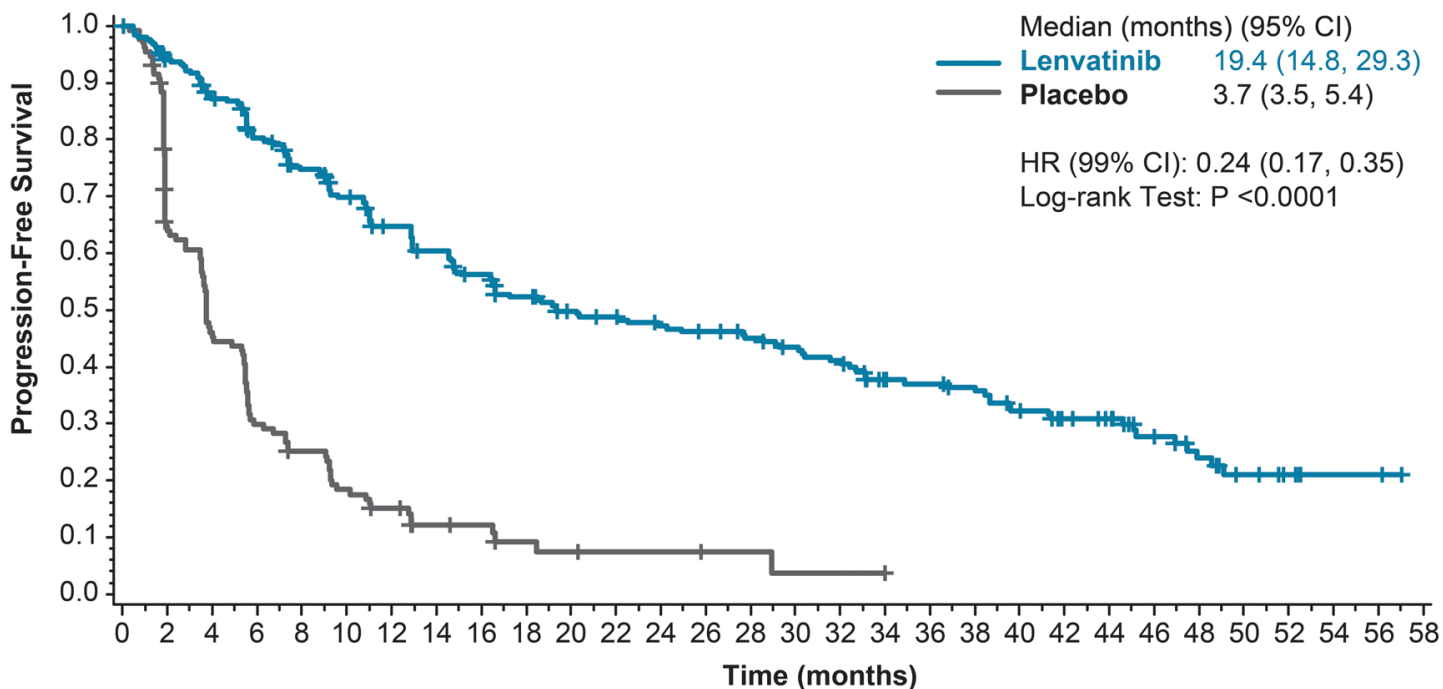

Number of subjects at risk:

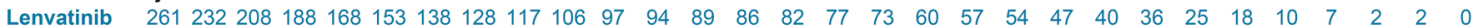
$\begin{array}{llllllllllllllllllllllllllllllll}\text { Placebo } & 131 & 79 & 57 & 37 & 30 & 22 & 17 & 9 & 8 & 5 & 4 & 3 & 3 & 2 & 2 & 1 & 1 & 1 & 0 & 0 & 0 & 0 & 0 & 0 & 0 & 0 & 0 & 0 & 0 & 0\end{array}$

\section{Figure 1}

Kaplan-Meier estimate of progression-free survival by treatment. Cl, confidence interval; HR, hazard ratio.

interesting variations by subgroup were observed; for example, the median DOR was inversely correlated with a smaller disease burden. Importantly, the DOR was similar among patients who had prior anti-VEGF therapy (25\%, $n=40$ ) and those who did not, which demonstrates the effectiveness of treatment with lenvatinib. Differences in median DOR reported in some subgroups should be explored more fully in adequately designed and powered studies.
This updated data analysis also confirmed that lenvatinib is associated with prolonged PFS compared with placebo (median 19.4 months vs 3.7 months; HR 0.24; 99\% CI, 0.17-0.35; nominal $P<0.0001$ ), a similar PFS benefit as observed in the primary analysis of the trial (18.3 vs 3.6 months). However, notably, the median PFS in those patients who respond to lenvatinib treatment with a complete or partial decrease in tumor size was prolonged to 33.1 months, further emphasizing the

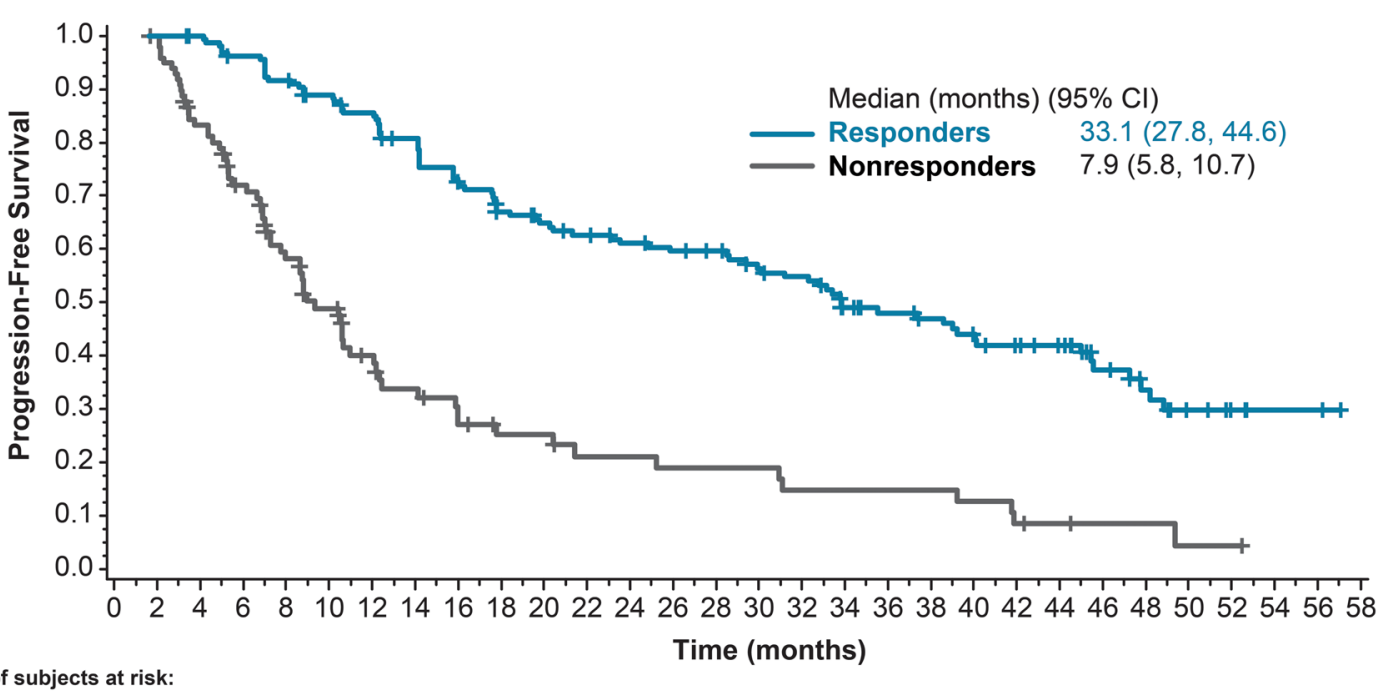

Number of subjects at risk:

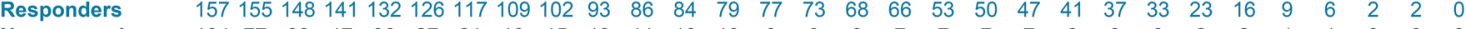
Nonresponders $\quad \begin{array}{lllllllllllllllllllllllllllllllllll} & 104 & 77 & 60 & 47 & 36 & 27 & 21 & 19 & 15 & 13 & 11 & 10 & 10 & 9 & 9 & 9 & 7 & 7 & 7 & 7 & 6 & 3 & 3 & 2 & 2 & 1 & 1 & 0 & 0 & 0\end{array}$

Figure 2

Kaplan-Meier estimate of progression-free survival for responders and non-responders. Cl, confidence interval. 
Table 2 Summary of tumor response per investigator assessment.

\begin{tabular}{l} 
Parameter \\
\hline Best overall response, $n(\%)$ \\
CR \\
PR \\
SD \\
Durable SD \\
PD \\
NE \\
Unknown \\
Objective response rate, $n(\%)$ \\
$95 \% \mathrm{Cl}$ \\
Median time to first objective response, months $(95 \% \mathrm{Cl})$ \\
DCR, $n(\%)$ \\
$95 \% \mathrm{Cl}$ \\
CBR, $n(\%)$ \\
$95 \% \mathrm{Cl}$ \\
Median duration of SD, months $(95 \% \mathrm{Cl})$
\end{tabular}

\begin{tabular}{c}
\hline Lenvatinib $(n=261)$ \\
$5(1.9)$ \\
$152(58.2)$ \\
$79(30.3)$ \\
$57(21.8)$ \\
$10(3.8)$ \\
$2(0.8)$ \\
$13(5.0)$ \\
$157(60.2)$ \\
$54.2-66.1$ \\
$3.5(1.9-3.7)$ \\
$236(90.4)$ \\
$86.9-94.0$ \\
$214(82.0)$ \\
$77.3-86.7$ \\
$9.6(7.6-14.8)$ \\
\hline
\end{tabular}

\begin{tabular}{c}
\hline Placebo $(n=131)$ \\
\hline $1(0.8)$ \\
$2(1.5)$ \\
$77(58.8)$ \\
$51(38.9)$ \\
$45(34.4)$ \\
$2(1.5)$ \\
$4(3.1)$ \\
$3(2.3)$ \\
$0.0-4.9$ \\
$9.4(1.8-11.0)$ \\
$80(61.1)$ \\
$52.7-69.4$ \\
$54(41.2)$ \\
$32.8-49.7$ \\
$5.7(5.5-7.4)$
\end{tabular}

Updated data, cutoff: 1 September 2016.

$\mathrm{CBR}$, clinical benefit rate; $\mathrm{Cl}$, confidence interval; $\mathrm{CR}$, complete response; $\mathrm{DCR}$, disease control rate; NE, not evaluable; PD, progressive disease; PR, partial response; $S D$, stable disease.

promising efficacy demonstrated by lenvatinib in this patient population.

The only other agent approved by the FDA for RR-DTC is sorafenib. The development of resistance to sorafenib is widely observed in patients with thyroid cancer who initially experienced a PR or SD, and, therefore, a plan for alternative treatments is required for these patients (Pitoia \& Jerkovich 2016). In this context, the durable response exhibited by patients in SELECT who received lenvatinib treatment, including those having failed prior anti-VEGF therapy, is especially important. The prolonged PFS and DOR observed with lenvatinib treatment may be due, in part, to lenvatinib's inhibition of multiple intracellular signaling targets not targeted by other VEGF inhibitors, including FGFR (St Bernard et al. 2005, Laursen et al. 2016). Activation of the FGFR pathway has been implicated in the development of resistance to VEGF-targeted therapies (Dieci et al. 2013), and, therefore, it is possible that lenvatinib-mediated inhibition of FGFRs 1-4 may play a role in the extended DOR exhibited by patients who responded to lenvatinib in SELECT.

In the primary analysis of SELECT, almost all patients in the lenvatinib treatment group experienced a treatment-related adverse event (Schlumberger et al. 2015), although most adverse events could be managed with dose modifications or medical therapy. We note that after 3 years of follow-up, the percentage of patients who experienced grade $\geq 3$ lenvatinib-related adverse events increased by less than $5 \%$, from $75.9 \%$ in the primary analysis to $80.8 \%$ in this analysis, with no new treatment-related deaths reported. This is consistent with a previous analysis of adverse events in SELECT, which concluded that most adverse events occurred early in the course of treatment (Haddad et al. 2017). This is important in the context of the prolonged DOR data showing that ongoing treatment with lenvatinib in those patients who demonstrate an initial response can result in a prolonged response to treatment. Thus, judicious management of adverse events in patients receiving lenvatinib long term is an especially important consideration for clinicians.

This analysis was limited by the lack of quality-of-life assessments. This prevents a complete evaluation of the benefits of prolonged lenvatinib treatment. Therefore, future studies of lenvatinib in this patient population should include quality-of-life assessments.

In conclusion, this updated analysis reinforced that lenvatinib treatment prolonged PFS compared with placebo in patients from SELECT with RR-DTC. Importantly, patients responding to lenvatinib demonstrated a prolonged PFS and DOR, suggesting that treatment with lenvatinib did not result in the same level of resistance as observed for some other TKIs. In addition, maintaining lenvatinib treatment by carefully managing adverse events can lead to a prolonged, durable response in $60.2 \%$ of patients. Further investigation is warranted to explore the considerations that must be made by clinicians treating patients with RR-DTC. 


\section{Declaration of interest}

Andrew $\mathrm{G}$ Gianoukakis reports grants and nonfinancial support from Eisai Inc. during the study. Corina E Dutcus and Matthew Guo are employees of Eisai, and Nicolas Batty and Mahadi Baig are former employees of Eisai.

\section{Funding}

This work was supported by Eisai Inc., Woodcliff Lake, NJ, USA.

\section{Acknowledgments}

The authors thank the patients and their families, as well as the investigators and their teams, who were involved in this study. They also thank Elton Mathias and Yaohua He for their valuable contributions to the analyses and discussions. Editorial assistance was provided by Oxford PharmaGenesis Inc., Newtown, PA, USA and was funded by Eisai Inc., Woodcliff Lake, NJ, USA

\section{References}

Brose MS, Nutting CM, Jarzab B, Elisei R, Siena S, Bastholt L, de la Fouchardiere C, Pacini F, Paschke R, Shong YK, et al. 2014 Sorafenib in radioactive iodine-refractory, locally advanced or metastatic differentiated thyroid cancer: a randomised, double-blind, phase 3 trial. Lancet 384 319-328. (https://doi.org/10.1016/S01406736(14)60421-9)

Busaidy NL \& Cabanillas ME 2012 Differentiated thyroid cancer: management of patients with radioiodine nonresponsive disease. Journal of Thyroid Research 2012 618985. (https://doi. org/10.1155/2012/618985)

Cooper DS, Doherty GM, Haugen BR, Kloos RT, Lee SL, Mandel SJ, Mazzaferri EL, McIver B, Pacini F, Schlumberger M, et al. 2009 Revised American Thyroid Association management guidelines for patients with thyroid nodules and differentiated thyroid cancer. Thyroid 19 1167-1214. (https://doi.org/10.1089/thy.2009.0110)

Dieci MV, Arnedos M, Andre F \& Soria JC 2013 Fibroblast growth factor receptor inhibitors as a cancer treatment: from a biologic rationale to medical perspectives. Cancer Discovery 3 264-279. (https://doi. org/10.1158/2159-8290.CD-12-0362)

Durante C, Haddy N, Baudin E, Leboulleux S, Hartl D, Travagli JP, Caillou B, Ricard M, Lumbroso JD, De Vathaire F, et al. 2006 Longterm outcome of 444 patients with distant metastases from papillary and follicular thyroid carcinoma: benefits and limits of radioiodine therapy. Journal of Clinical Endocrinology and Metabolism 91 2892-2899. (https://doi.org/10.1210/jc.2005-2838)

Haddad RI, Schlumberger M, Wirth LJ, Sherman EJ, Shah MH, Robinson B, Dutcus CE, Teng A, Gianoukakis AG \& Sherman SI 2017 Incidence and timing of common adverse events in Lenvatinibtreated patients from the SELECT trial and their association with survival outcomes. Endocrine 56 121-128. (https://doi.org/10.1007/ s12020-017-1233-5)
Laursen R, Wehland M, Kopp S, Pietsch J, Infanger M, Grosse J \& Grimm D 2016 Effects and role of multikinase inhibitors in thyroid cancer. Current Pharmaceutical Design 22 5915-5926. (https://doi.org/ $10.2174 / 1381612822666160614084943)$

Matsui J, Funahashi Y, Uenaka T, Watanabe T, Tsuruoka A \& Asada M 2008a Multi-kinase inhibitor E7080 suppresses lymph node and lung metastases of human mammary breast tumor MDA-MB-231 via inhibition of vascular endothelial growth factor-receptor (VEGF-R) 2 and VEGF-R3 kinase. Clinical Cancer Research 14 5459-5465. (https:// doi.org/10.1158/1078-0432.CCR-07-5270)

Matsui J, Yamamoto Y, Funahashi Y, Tsuruoka A, Watanabe T, Wakabayashi T, Uenaka T \& Asada M 2008b E7080, a novel inhibitor that targets multiple kinases, has potent antitumor activities against stem cell factor producing human small cell lung cancer H146, based on angiogenesis inhibition. International Journal of Cancer 122 664-671. (https://doi.org/10.1002/ijc.23131)

Okamoto K, Kodama K, Takase K, Sugi NH, Yamamoto Y, Iwata M \& Tsuruoka A 2013 Antitumor activities of the targeted multi-tyrosine kinase inhibitor lenvatinib (E7080) against RET gene fusion-driven tumor models. Cancer Letters 340 97-103. (https://doi.org/10.1016/j. canlet.2013.07.007)

Pacini F, Ito Y, Luster M, Pitoia F, Robinson B \& Wirth L 2012 Radioactive iodine-refractory differentiated thyroid cancer: unmet needs and future directions. Expert Review of Endocrinology and Metabolism 7 541-554. (https://doi.org/10.1586/eem.12.36)

Pitoia F \& Jerkovich F 2016 Selective use of sorafenib in the treatment of thyroid cancer. Drug Design, Development and Therapy 10 1119-1131. (https://doi.org/10.2147/DDDT.S82972)

Schlumberger M, Tahara M, Wirth LJ, Robinson B, Brose MS, Elisei R, Habra MA, Newbold K, Shah MH, Hoff AO, et al. 2015 Lenvatinib versus placebo in radioiodine-refractory thyroid cancer. New England Journal of Medicine 372 621-630. (https://doi.org/10.1056/ NEJMoa1406470)

St Bernard R, Zheng L, Liu W, Winer D, Asa SL \& Ezzat S 2005 Fibroblast growth factor receptors as molecular targets in thyroid carcinoma. Endocrinology 146 1145-1153. (https://doi.org/10.1210/ en.2004-1134)

Tohyama O, Matsui J, Kodama K, Hata-Sugi N, Kimura T, Okamoto K, Minoshima Y, Iwata M \& Funahashi Y 2014 Antitumor activity of lenvatinib (e7080): an angiogenesis inhibitor that targets multiple receptor tyrosine kinases in preclinical human thyroid cancer models. Journal of Thyroid Research 2014 638747. (https://doi. org/10.1155/2014/638747)

Worden F 2014 Treatment strategies for radioactive iodine-refractory differentiated thyroid cancer. Therapeutic Advances in Medical Oncology 6 267-279. (https://doi.org/10.1177/1758834014548188)

Xing M, Haugen BR \& Schlumberger M 2013 Progress in molecularbased management of differentiated thyroid cancer. Lancet $\mathbf{3 8 1}$ 1058-1069. (https://doi.org/10.1016/S0140-6736(13)60109-9)

Yamamoto Y, Matsui J, Matsushima T, Obaishi H, Miyazaki K, Nakamura K, Tohyama O, Semba T, Yamaguchi A, Hoshi SS, et al. 2014 Lenvatinib, an angiogenesis inhibitor targeting VEGFR/FGFR, shows broad antitumor activity in human tumor xenograft models associated with microvessel density and pericyte coverage. Vascular Cell 6 18. (https://doi.org/10.1186/2045-824X-6-18)

Received in final form 9 April 2018

Accepted 17 April 2018
(C) 2018 The authors Published by Bioscientifica Ltd. Printed in Great Britain

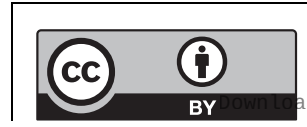

This work is licensed under a Creative Commons Attribution 4.0 International License. ded from Bioscientifica.com at 04/26/2023 12:41:55PM via free access 